\title{
Impact of the coronavirus disease pandemic on cancer care in Croatia: a multicentre cross-sectional study
}

\author{
Renata Kelemenic-Drazin ${ }^{1}$, Anuska Budisavljevic ${ }^{2}$, Natalija Dedic Plavetic ${ }^{3}$, Iva Kardum Fucak ${ }^{4}$, Tajana Silovski ${ }^{3}$, Vesna Telesmanic Dobric ${ }^{5}$, \\ Mario Nalbani ${ }^{6}$, Zvonimir Curic ${ }^{6}$, Zvjezdana Boric-Mikez ${ }^{7}$, Tatjana Ladenhauser ${ }^{8}$, Dragan Trivanovic ${ }^{2}$, Zeljko Vojnovic ${ }^{1}$, Ilijan Tomas ${ }^{8}$ \\ and Stjepko Plestina ${ }^{3}$ \\ ${ }^{1}$ Department of Haematology, Oncology and Clinical Immunology, General Hospital Varazdin, I. Mestrovica 1, 42000, Varazdin, Croatia \\ ${ }^{2}$ Department of Haematology and Oncology, General Hospital Pula, Zagrebacka 30, 52100, Pula, Croatia \\ ${ }^{3}$ Department of Oncology, University Hospital Centre Zagreb, Kispaticeva 12, 10000, Zagreb, Croatia \\ ${ }^{4}$ Department of Gastroenterology, Haematology and Oncology, General Hospital Dr. Tomislav Bardek Koprivnica, Ulica doktora Zeljka Selingera bb, 48000, \\ Koprivnica, Croatia \\ ${ }^{5}$ Department of Oncology and Nuclear Medicine, General Hospital Zadar, Ul. Boze Pericica 5, 23000, Zadar, Croatia \\ ${ }^{6}$ Department of Oncology, General Hospital Dubrovnik, Dr. Roka Misetica 2, 20000, Dubrovnik, Croatia \\ ${ }^{7}$ Department of Haematology and Oncology, General Hospital Dr. Josip Bencevic Slavonski Brod, Ul. Andrije Stampara, 35000, Slavonski Brod, Croatia \\ ${ }^{8}$ Department of Oncology, University Hospital Centre Osijek, Ul. Josipa Huttlera 4, 31000, Osijek, Croatia
}

\section{Abstract}

Purpose: The coronavirus disease (COVID-19) pandemic has greatly affected the oncology community worldwide. Lockdowns, an epidemiological measure, have made it difficult for oncologists to provide care. In this study, we analysed the impact of the COVID19 pandemic on Croatian cancer care.

Methods: This was a multicentre cross-sectional observational study of 422 patients who received systemic oncology therapy during the pandemic. The patients completed a survey to capture their views on the impact of the COVID-19 pandemic on their cancer care. Univariate descriptive and bivariate analyses were performed to analyse the relationship between the patients' perspective on the impact of the COVID-19 pandemic on cancer care and the quality of Croatian cancer care and their clinical and sociodemographic data.

Results: Discontinuation or change in cancer treatment during the COVID-19 pandemic was observed in $10.2 \%$ of cases. Most did not change their place of treatment owing to the lockdown (97.6\%). $14.7 \%$ of the patients felt that the quality of cancer care received had changed during the pandemic.

Conclusions: In the first few months of the pandemic, Croatia had a favourable epidemiological situation. However, $25 \%$ of patients with cancer reported that the pandemic affected cancer treatment and the quality of cancer care.

Keywords: cancer care, coronavirus, COVID-19, pandemic, patients' perspective

Correspondence to: Renata Kelemenic-Drazin Email: renata.kelemenicdrazin@optinet.hr

ecancer 2021, 15:1263

https://doi.org/10.3332/ecancer.2021.1263

Published: 07/07/2021

Received: 24/03/2021

Publication costs for this article were supported by ecancer (UK Charity number 1176307).

Copyright: (c) the authors; licensee ecancermedicalscience. This is an Open Access article distributed under the terms of the Creative Commons Attribution License (http:// creativecommons.org/licenses/by/4.0), which permits unrestricted use, distribution, and reproduction in any medium, provided the original work is properly cited. 


\section{Background}

The coronavirus disease (COVID-19) pandemic has greatly affected the oncology community and the wider health care system.

The first report on COVID-19 in patients with cancer was published in The Lancet Oncology in February 2020 [1] and suggests that cancer patients have a higher risk of COVID-19 and a poorer prognosis than non-cancer patients. Although initial opinions were that there was insufficient evidence to establish an association between cancer and COVID-19 [2, 3], new findings suggest that the presence of cancer significantly affects the mortality rate in patients with COVID-19 [4, 5].

To prevent the spread of the infection, the lockdown has been introduced. Therefore, oncology patients may not receive all of the necessary medical services, from hospital arrival to adequate systemic or local oncology therapy. This problem has become evident in Croatia, wherein 4.1 million inhabitants live in 20 counties and the capital, Zagreb. Seven of the 20 counties (with $18 \%$ of the total population) do not have organised cancer care facilities, and oncology patients in these counties are forced to travel to other counties daily to receive appropriate oncology treatment [6-8]. That could interrupt the continuity of oncology care and increase the risk of inadequate and delayed care during the COVID-19 pandemic and ultimately lead to poorer treatment outcomes $[9,10]$.

On 25 February 2020, the first case of COVID-19 in Croatia was reported in Zagreb [8]. The World Health Organization officially declared COVID-19 as a global pandemic on 11 March 2020 and called on governments to take urgent action [11].

By 24 May 2020 (date of completion of the current study), of the 2,244 people with COVID-19 in Croatia, 2,027 had recovered, 99 had died and 118 were still receiving treatment [8]. Considering these numbers compared with the 1 million inhabitants in Croatia, by the end of the study, 549 people were infected, and 24 patients had died. According to the number of deaths per million inhabitants, Croatia was among the five best-positioned countries in Europe [12-14].

This study aimed to determine the impact of the COVID-19 pandemic on cancer care and treatment in Croatia, as assessed by the patients that received treatment. We also investigated whether patients experienced a change in oncology treatment (change in treatment protocol or schedule), discontinuation of treatment or change in the treatment site. Furthermore, we also assessed whether the participants perceived that the quality of cancer care received during the pandemic had changed.

\section{Methods}

\section{Study design and participants}

An academic cross-sectional multicentre observational study was conducted between 20 April and 24 May 2020 (in the second and third months of the pandemic in Croatia), in eight oncology centres in Croatia: two university hospitals (UH) and six general/county hospitals (GH). This study included 422 patients and was initiated and conducted by the Council of Institutions of the Croatian Society for Medical Oncology, a collaborating society of the European Society for Medical Oncology.

The patients included in the study were older than 18 years, had already started systemic oncology treatment before 25 February 2020 (date of the first patient with COVID-19 reported in Croatia), were newly diagnosed with cancer and received oncology treatment during the pandemic. The study included patients with different stages of malignancy. The study included patients with early-stage cancers, whether treated with adjuvant or neoadjuvant therapy, as well as patients with disseminated malignancies treated in a metastatic, palliative setting.

We used a self-reported questionnaire entitled 'The impact of the COVID-19 pandemic on the treatment of cancer patients in the Republic of Croatia'. Content validity of the survey was assessed by presenting it to a group of five oncologists. The obtained final version of the survey was further piloted on a sample of patients with cancer in different institutions $(n=20)$ to ensure that all the items used were clear and understandable. This questionnaire was approved by the Ethics Committee of the hospitals where it was conducted. All participants provided written informed consent for voluntarily participating in the study. No personal data were collected. 
The questions in the first part of the survey collected data on the sociodemographic characteristics of patients, treating institution (UH or $\mathrm{GH}$ ) and the distance the hospital was from the patient's home. We divided patients into three groups according to the distance to the hospital (less than $50 \mathrm{~km}, 50-100 \mathrm{~km}$, more than $100 \mathrm{~km}$ ), education level and household income. Using data from the Croatian Central Bureau of Statistics [15], we divided patients into groups according to income. Patients with an average level of annual household income were included in the medium-income group, while the other patients were included in the low- or high-income groups (depending on whether their incomes were below or above the average level of annual household income in Croatia). Regarding the education level, we divided patients into the elementary school group (basic or primary), upper secondary school group (high school) and tertiary education group (undergraduate or postgraduate) [7]. We also divided patients according to their marital status (unmarried, divorced, widowed, in partnership (married or in a de facto relationship)).

The second part of the survey focussed on collecting data on the patients' cancer and included questions on the cancer site, stage of disease (non-metastatic or metastatic cancer), duration of cancer treatment (less than 6 months, 6-12 months or longer than 12 months) and type of cancer therapy (chemotherapy, targeted/immunotherapy or a combination of different types of therapies: chemotherapy and targeted therapy, hormonal therapy and targeted therapy, immunotherapy and chemotherapy). Regarding the cancer site, patients were grouped as having breast cancer, gastrointestinal cancer (colorectal, pancreatic, gastric, oesophagus, gallbladder and bile duct cancer), genitourinary cancer (prostate, kidney and bladder cancer), lung cancer, other sites (sarcomas, melanomas and head and neck cancer) and gynaecological cancer (ovarian and endometrial cancer).

In the third part of the survey, we determined whether, entirely due to the pandemic, the patients' cancer treatment discontinued or changed and whether they perceived a change in the quality of cancer care received (quality of cancer treatment or quality of cancer diagnosis). The change in treatment referred to a change in the place of treatment (referral of the patient to the nearest oncology centre) or a change in the treatment schedule (such as replacing 2-week or 1-week cycles of therapy with 3-week ones) or type of therapy (such as changing parenteral therapies to oral ones). This did not apply to patients for whom treatment was discontinued or changed due to the progression of malignancy. The total number of completed questionnaires was 422 .

\section{Statistical analysis}

Univariate descriptive analyses were carried out to describe the data. The Kolmogorov-Smirnov test was used to test the normality of data distribution. For descriptive analysis, continuous variables were presented as the median with an interquartile range (IQR). Categorical variables were presented as a number (\%). A bivariate analysis, chi-square test for categorical data and Fisher's exact test were used to study the relationship between patients' perspectives on the impact of the COVID-19 pandemic on oncology treatment as well as the relationship between changes in the quality of cancer treatment and diagnostics, and other clinical and sociodemographic data. During the post hoc analysis, Bonferroni's correction was used to control for Type I error.

All statistical analyses were carried out using SPSS Statistics, version 20.0. A two-sided $p$-value of less than 0.05 was considered to indicate statistical significance.

\section{Results}

\section{Demographic and clinical characteristics}

The demographic and clinical features of this cohort are summarised in Table 1. The median patient age was 60.0 years. Most of the respondents were female ( $n=283,67.1 \%)$, and the majority of the patients had low- $(n=191,45.3 \%)$ and medium-income levels $(n=164,38.9 \%)$ according to the Croatian average. Most patients had completed high school $(n=257,60.9 \%)$, and $71.1 \%(n=300)$ of the respondents lived with a partner (either married or in a de facto relationship). 
Table 1. Demographic and baseline clinical characteristics of the patients.

\begin{tabular}{|c|c|}
\hline Characteristic & Patients $(N=422)$ \\
\hline Median age (IQR), years & $60.0(18) 18-82$ \\
\hline \multicolumn{2}{|l|}{ Sex } \\
\hline Male & 139 (32.9) \\
\hline Female & $283(67.1)$ \\
\hline \multicolumn{2}{|l|}{ Household income } \\
\hline Low & $191(45.3)$ \\
\hline Medium & 164 (38.9) \\
\hline High & $67(15.9)$ \\
\hline \multicolumn{2}{|l|}{ Education level } \\
\hline Primary education & $67(15.9)$ \\
\hline Secondary education & $257(60.9)$ \\
\hline Tertiary education & $98(23.2)$ \\
\hline \multicolumn{2}{|l|}{ Partnership status } \\
\hline In partnership (married or de facto) & $300(71.1)$ \\
\hline Single, divorced, widowed & $122(28.9)$ \\
\hline \multicolumn{2}{|l|}{ Tumour diagnosis } \\
\hline Breast cancer & $201(47.6)$ \\
\hline Gastrointestinal cancer & $133(31.5)$ \\
\hline Genitourinary cancer & $30(7.1)$ \\
\hline Lung cancer & $22(5.2)$ \\
\hline Other & $19(4.5)$ \\
\hline Gynaecology cancer & $17(4.0)$ \\
\hline \multicolumn{2}{|l|}{ Tumour stage } \\
\hline Non-metastatic cancer & $192(45.5)$ \\
\hline Metastatic cancer & $230(54.5)$ \\
\hline \multicolumn{2}{|l|}{ Duration of disease } \\
\hline 1-6 months & $133(31.5)$ \\
\hline 6-12 months & $87(20.6)$ \\
\hline More than 12 months & $202(47.9)$ \\
\hline \multicolumn{2}{|l|}{ Types of treatment } \\
\hline Chemotherapy & $250(59.2)$ \\
\hline Target/immunotherapy & $101(23.9)$ \\
\hline Combination of different therapies & $71(16.8)$ \\
\hline \multicolumn{2}{|l|}{ Hospital } \\
\hline $\mathrm{UH}$ & 142 (33.6) \\
\hline $\mathrm{GH}$ & $280(66.4)$ \\
\hline
\end{tabular}


Table 1. Demographic and baseline clinical characteristics of the patients. (Continued)

\begin{tabular}{|l|c|}
\hline \multicolumn{2}{|l|}{ Distance to the hospital } \\
\hline Less than $50 \mathrm{~km}$ & $355(84.1)$ \\
\hline $50-100 \mathrm{~km}$ & $33(7.8)$ \\
\hline More than $100 \mathrm{~km}$ & $34(8.1)$ \\
\hline
\end{tabular}

Data are presented as $n$ (\%) unless noted otherwise

Breast cancer was the most common type of cancer $(n=201,47.6 \%)$, followed by gastrointestinal cancer $(n=133,31.5 \%)$. The other tumour sites were less common. The majority of the patients $(n=230,54.5 \%)$ were treated for metastatic cancer.

Almost half of the respondents were treated for more than 12 months ( $n=202,47.9 \%$ ), and 59.2\% $(n=250)$ were treated with chemotherapy.

Furthermore, $66.4 \%(n=280)$ of patients were treated in a $\mathrm{GH}$, and most patients $(n=355,84.1 \%)$ lived less than $50 \mathrm{~km}$ from the hospital.

\section{Patients' perspective on the impact of the COVID-19 pandemic on cancer care}

\section{Discontinuation of treatment or change in treatment}

The results of our study showed that, during the COVID-19 pandemic, $10.2 \%$ of patients $(n=43)$ had their oncology treatment discontinued or changed (Tables 2-5).

Among the patients who discontinued treatment $(n=19,4.5 \%), 63 \%(n=12)$ discontinued treatment on the recommendation of their oncologist, and $37 \%(n=7)$ discontinued treatment themselves. The most common reasons for oncologists to recommend discontinuing treatment were higher risk of COVID-19 (91.7\%) and a higher risk of serious complications of COVID-19 (8.3\%). The most common reasons why patients decided to discontinue treatment were pandemic fear (28.6\%), inability to come to the hospital (57.1\%) and self-isolation (14.3\%). The majority of the patients ( $n=18$ of 19 patients, $94.7 \%$ ) were satisfied with the information received from oncologists regarding treatment discontinuation, and 13 (68.4\%) of 19 patients felt that the discontinuation of treatment would not affect their disease course or prognosis.

To check for any differences between the patients who discontinued treatment and those who did not discontinue, we performed a chisquare test using sex, age, partnership status, disease site, disease stage and distance to the hospital and a Fisher's exact test using household income and education level (Table 2). Patients were divided into groups according to the disease site: breast cancer, colorectal cancer and others. The 'others' category included all other disease sites due to their low frequency.

There was a significant correlation between treatment discontinuation and the distance to the hospital $(p<0.05)$. This indicates that patients who resided more than $50 \mathrm{~km}$ from the hospital were more likely to discontinue treatment.

There was no significant correlation between treatment discontinuation and sex $(p=0.711)$, age $(p=0.572)$, household income $(p=0.267)$, educational degree $(p=1.00)$, partnership status $(p=0.799)$, disease site $(p=0.355)$ or disease stage $(p=0.438)$.

In patients who had a change in treatment ( $n=24$ of 422 patients, $5.7 \%$ ), the most common change was in the treatment schedule (58.3\%). Other reasons for treatment changes were as follows: change in the type of therapy $(29.2 \%)$, long waiting time for the start of chemotherapy treatment (4.2\%) and delayed surgery (4.2\%).

The change in treatment was most often recommended by an oncologist ( $n=21$ of 24 patients, $87.5 \%$ ) due to a higher risk of COVID-19 (66.7\%), higher risk of serious complications of COVID-19 (4.8\%), comorbidity (4.8\%), side effects of treatment (9.5\%), additional tests (4.8\%) and inability to come to the hospital (4.8\%). Treatment was changed at the request of three patients ( $n=3$ of 24 patients, $12.5 \%$ ) due to pandemic fear (66.7\%) and problems with diagnostic processing (33.3\%). All patients who had a change in treatment $(n=24,100 \%)$ were satisfied with the information they received from their oncologist, and most patients ( $n=17$ of 24 patients, $70.8 \%$ ) thought that the change in treatment would not affect the course of their disease and prognosis. 
Table 2. Relationship between the discontinuation of treatment and sociodemographic and clinical data $(N=19)$.

\begin{tabular}{|c|c|c|}
\hline Characteristics & \multicolumn{2}{|c|}{$p$-value } \\
\hline Sex & & $0.711^{\mathrm{a}}$ \\
\hline Male & $7(1.7)$ & \\
\hline Female & $12(2.8)$ & \\
\hline Age & & $0.572^{\mathrm{a}}$ \\
\hline Less than 55 years & $8(1.9)$ & \\
\hline $55+$ years & $11(2.6)$ & \\
\hline Household income & & $0.267^{b}$ \\
\hline Low & $12(2.8)$ & \\
\hline Medium & $6(1.4)$ & \\
\hline High & $1(0.2)$ & \\
\hline Education level & & $1.00^{b}$ \\
\hline Elementary & $3(0.7)$ & \\
\hline Secondary & $12(2.8)$ & \\
\hline Undergraduate and postgraduate & $4(0.9)$ & \\
\hline Partnership status & & $0.799^{\mathrm{a}}$ \\
\hline In partnership & $14(3.3)$ & \\
\hline Single & $5(1.2)$ & \\
\hline Tumour diagnosis & & $0.355^{\mathrm{a}}$ \\
\hline Breast cancer & $8(1.9)$ & \\
\hline Colorectal cancer & $7(1.7)$ & \\
\hline Other & $4(0.9)$ & \\
\hline Tumour stage & & $0.438^{\mathrm{a}}$ \\
\hline Non-metastatic & $7(1.7)$ & \\
\hline Metastatic & $12(2.8)$ & \\
\hline Distance to the hospital & & $<0.050^{\mathrm{a} *}$ \\
\hline Less than $50 \mathrm{~km}$ & $12(2.8)$ & \\
\hline More than $50 \mathrm{~km}$ & $7(1.7)$ & \\
\hline
\end{tabular}

Data are presented as $n(\%)$ unless noted otherwise

a $p$-value calculated using a chi-square test

${ }^{\mathrm{b}} p$-value calculated using Fisher's exact test

*A two-sided asymptotic $p$-value of $<0.05$ was considered to indicate statistical significance

The chi-square test did not show any significant correlation between treatment change and sex $(p=0.966)$, age (less or more than 55 years, $p=0.247)$, partnership status ( $p=0.664)$, disease site (breast cancer, colorectal cancer, $p=0.762)$, disease stage $(p=0.973)$ and distance to the hospital $(p=0.913)$. The Fisher's exact test did not show a significant correlation between treatment change and household income $(p=$ $0.847)$, or educational degree $(p=0.914$; Table 3$)$. 
Table 3. Relationship between the change in treatment and sociodemographic and clinical data $(N=24)$.

\begin{tabular}{|c|c|c|}
\hline Characteristics & & \\
\hline Sex & & $0.966^{a}$ \\
\hline Male & $8(1.9)$ & \\
\hline Female & $16(3.8)$ & \\
\hline Age & & $0.247^{\mathrm{a}}$ \\
\hline Less than 55 years & $6(1.4)$ & \\
\hline $55+$ years & $18(4.3)$ & \\
\hline Household income & & $0.847^{b}$ \\
\hline Low & $12(2.8)$ & \\
\hline Medium & $8(1.9)$ & \\
\hline High & $4(0.9)$ & \\
\hline Education level & & $0.914^{b}$ \\
\hline Elementary & $4(0.9)$ & \\
\hline Secondary & $14(3.3)$ & \\
\hline Undergraduate and postgraduate & $6(1.4)$ & \\
\hline Partnership status & & $0.664^{a}$ \\
\hline In partnership & $18(4.3)$ & \\
\hline Single & $6(1.4)$ & \\
\hline Tumour diagnosis & & $0.762^{\mathrm{a}}$ \\
\hline Breast cancer & $10(2.4)$ & \\
\hline Colorectal cancer & $7(1.7)$ & \\
\hline Others & $7(1.7)$ & \\
\hline Tumour stage & & $0.973^{\mathrm{a}}$ \\
\hline Non-metastatic & $11(2.6)$ & \\
\hline Metastatic & $13(3.1)$ & \\
\hline Distance to the hospital & & $0.913^{\mathrm{a}}$ \\
\hline Less than $50 \mathrm{~km}$ & $20(4.7)$ & \\
\hline More than $50 \mathrm{~km}$ & $4(0.9)$ & \\
\hline
\end{tabular}

Data are presented as $n(\%)$ unless noted otherwise

${ }^{a} p$-value calculated using a chi-square test

${ }^{\mathrm{b}} p$-value calculated using Fisher's exact test

During the COVID-19 pandemic, most patients did not change their place of treatment due to the lockdown ( $n=412,97.6 \%)$. Patients who changed their place of treatment most often $(n=9)$ replaced the treatment at a UH with treatment at a GH/County Hospital on the recommendation of their oncologist. All patients (100\%) who changed their place of treatment expressed satisfaction with the oncologists' communication regarding the change. Most patients $(n=9)$ believed that a change in treatment place would not affect the course of their disease or prognosis. 


\section{Change in the quality of treatment and diagnostics}

Most patients did not experience a change in the quality of cancer treatment during the pandemic ( $n=395,93.6 \%$ of 422 patients). Moreover, some patients $(n=19,4.5 \%)$ felt that there was an improvement in the quality of oncology care, probably due to a smaller influx of nononcology patients into health facilities during the pandemic. In comparison, a smaller number of patients $(n=8,1.9 \%)$ considered that the quality of their treatment had deteriorated.

A significant correlation was found between sex, household income and the disease site and changes in oncology treatment quality $(p<0.05)$. Males and low-income patients were more likely to have perceived a change in the quality of their treatment. The quality of treatment was more likely to remain the same in patients with breast cancer. However, a marginally significant correlation was obtained between age and change in treatment quality $(p=0.050)$. We assume that this finding may indicate that a significant difference in the population still exists. This could probably be proven with a larger sample. There was no significant difference between the change in the quality of treatment and education level $(p<0.076)$, partnership status $(p<0.661)$, stage of the disease $(p=0.608)$ or distance to the hospital $(p=0.140$; Table 4$)$.

In addition to changes in the quality of treatment, we were also interested in whether there was a change in the quality of diagnostic processing during the pandemic. Thirty-five patients (8.3\%) reported a change in diagnostic quality (Table 5). There was no significant correlation between the change in the quality of diagnostic processing and sex $(p=0.353)$, age $(p=0.608)$, household income $(p=0.186)$, education level $(p<0.222)$, partnership status $(p<0.963)$, disease site $(p=0.739)$, disease stage $(p=0.276)$ or distance to the hospital $(p=0.096)$.

However, we found that $14.7 \%(n=62)$ of patients reported a change in the quality of cancer care due to the pandemic.

\section{Discussion}

In the first months of the COVID-19 pandemic, Croatia had a favourable epidemiological situation [8].

Accordingly, $10.2 \%$ of patients had an interruption or change in their oncological treatment. In most patients, the reason for this change was on the recommendation of an oncologist owing to the possible higher risk of COVID-19 and a higher risk of serious complications. The most common change in treatment was related to a change in the treatment schedule regardless of clinical or sociodemographic data and the distance to the hospital. But patients who resided more than $50 \mathrm{~km}$ away from the hospital were more likely to discontinue treatment regardless of their sociodemographic or clinical data.

A smaller number of patients in our study changed the treatment site due to the lockdown $(n=10,2.4 \%)$. They generally replaced the treatment at a University with a County Hospital, which is usually closer to where patients live.

Most of the patients did not experience a change in the quality of cancer treatment during the pandemic ( $n=395$ of 422 patients, $93.6 \%$ ). Our study showed that, in patients with breast cancer, the quality of treatment was more likely to remain the same. Males and low-income patients were more likely to report a change in treatment quality. $8.3 \%$ patients $(n=35)$ reported a change in diagnostic quality regardless of their clinical or sociodemographic data and distance to the hospital. In summary, 62 patients (14.7\%) reported a change in the quality of cancer care during the pandemic.

The first study on patients' perspectives on the influence of the COVID-19 pandemic on cancer treatment, published by Indian authors, showed that cancer patients from India wish to continue chemotherapy during the pandemic and are more worried about cancer progression than the COVID-19 [16]. A study by Dutch authors showed that, in the Netherlands, 30\% of cancer patients experienced changes to their oncology treatment or follow-up [17]. The U.S. study showed a 20\%-30\% reduction in oncology products during the COVID-19 pandemic, while a study by Italian authors confirmed the pandemic's impact on cancer care, but also that cancer patients are usually strongly motivated to continue cancer treatment despite the pandemic $[18,19]$.

This study has several strengths. Patients from all counties of Croatia were included. These counties differ in terms of the local epidemiological situation related to COVID-19. Furthermore, the study included 8 of the 13 oncology centres in Croatia, of which two are oncology clinical centres, and six are non-clinical oncology centres, therapy increasing the multicentricity. Furthermore, the respondents were all anonymous. 
Table 4. Relationship between the change in the quality of treatment and sociodemographic and clinical data $(N=27)$.

\begin{tabular}{|c|c|c|}
\hline Characteristic & \multicolumn{2}{|c|}{$p$-value } \\
\hline Sex & & $<0.050^{\mathrm{a} *}$ \\
\hline Male & $15(3.6)$ & \\
\hline Female & $12(2.8)$ & \\
\hline Age & & $0.050^{\mathrm{a}}$ \\
\hline Less than 55 years & $5(1.2)$ & \\
\hline $55+$ years & $22(5.2)$ & \\
\hline Household income & & $<0.050^{\mathrm{b} * *}$ \\
\hline Low & $19(4.5)$ & \\
\hline Medium & $5(1.2)$ & \\
\hline High & $3(0.7)$ & \\
\hline Education level & & $0.076^{\mathrm{b}}$ \\
\hline Elementary & $8(1.9)$ & \\
\hline Secondary & $16(3.8)$ & \\
\hline Undergraduate and postgraduate & $3(0.7)$ & \\
\hline Partnership status & & $0.661^{a}$ \\
\hline In partnership & $18(4.3)$ & \\
\hline Single & $9(2.1)$ & \\
\hline Tumour diagnosis & & $<0.050^{\mathrm{a} *}$ \\
\hline Breast cancer & $6(1.4)$ & \\
\hline Colorectal cancer & $8(1.9)$ & \\
\hline Others & $13(3.1)$ & \\
\hline Tumour stage & & $0.608^{\mathrm{a}}$ \\
\hline Non-metastatic & $11(2.6)$ & \\
\hline Metastatic & $16(3.8)$ & \\
\hline Distance to the hospital & & $0.140^{a}$ \\
\hline Less than $50 \mathrm{~km}$ & $20(4.7)$ & \\
\hline More than $50 \mathrm{~km}$ & $7(1.7)$ & \\
\hline
\end{tabular}

Data are presented as $n(\%)$ unless noted otherwise

${ }^{a} p$-value calculated using a chi-square test

${ }^{\mathrm{b}} \mathrm{P}$-value calculated using Fisher's exact test

*A two-sided asymptotic $p<0.05$ was considered to indicate statistical significance

${ }^{* *}$ A two-sided exact $p<0.05$ was considered to indicate statistical significance 
Table 5. Relationship between changes in the quality of cancer diagnostic and sociodemographic and clinical data $(N=35)$.

\begin{tabular}{|c|c|c|}
\hline Characteristic & \multicolumn{2}{|c|}{$p$-value } \\
\hline Sex & & $0.353^{\mathrm{a}}$ \\
\hline Male & $14(3.3)$ & \\
\hline Female & $21(5.0)$ & \\
\hline Age & & $0.608^{a}$ \\
\hline Less than 55 years & $14(3.3)$ & \\
\hline $55+$ years & $21(5.0)$ & \\
\hline Household income & & $0.186^{a}$ \\
\hline Low & $16(3.8)$ & \\
\hline Medium & $10(2.4)$ & \\
\hline High & $9(2.1)$ & \\
\hline Education level & & $0.222^{a}$ \\
\hline Elementary & $3(0.7)$ & \\
\hline Secondary & $26(6.2)$ & \\
\hline Undergraduate and postgraduate & $6(1.4)$ & \\
\hline Partnership status & & $0.963^{\mathrm{a}}$ \\
\hline In partnership & $25(5.9)$ & \\
\hline Single & $10(2.4)$ & \\
\hline Tumour diagnosis & & $0.739^{a}$ \\
\hline Breast cancer & $16(3.8)$ & \\
\hline Colorectal cancer & $7(1.7)$ & \\
\hline Others & $12(2.8)$ & \\
\hline Tumour stage & & $0.276^{\mathrm{a}}$ \\
\hline Non-metastatic & $19(4.5)$ & \\
\hline Metastatic & $16(3.8)$ & \\
\hline Distance to the hospital & & $0.096^{a}$ \\
\hline Less than $50 \mathrm{~km}$ & $26(6.2)$ & \\
\hline More than $50 \mathrm{~km}$ & $9(2.1)$ & \\
\hline
\end{tabular}

Data are presented as $n(\%)$ unless noted otherwise

${ }^{a} p$-value calculated using a chi-square test

${ }^{\mathrm{b}} p$-value calculated using Fisher's exact test

This study also has several limitations. First, this study did not include patients who did not present at health facilities as planned during the survey period. These patients may not have presented due to objective reasons (issues with transportation due to lockdown, distance from the place of treatment, unable to arrange an escort) or subjective reasons such as pandemic fear. The research was conducted only in the first wave of the COVID-19 pandemic in Croatia, and due to the pandemic of an unknown virus, as well as due to lockdown, the reactions of patients could have been more intense. Although the study included patients from all counties in Croatia, this study did not include the 
oncology centre of Split-Dalmatia County, where 11\% of the population lives [8] and which had the most unfavourable epidemiological situation in Croatia in terms of the number of people infected and the number of deaths.

The COVID-19 pandemic is still ongoing, and despite many efforts, the ideal treatment approach for patients with cancer during the COVID19 pandemic is still unclear. Due to the overload of health systems, there is a real danger that oncology care will become unavailable in some parts of the world [20].

\section{Conclusion}

In the first few months of the pandemic, Croatia had a favourable epidemiological situation. However, $25 \%$ of patients with cancer reported that the pandemic affected cancer treatment and the quality of cancer care.

\section{Conflicts of interest}

All authors have declared no conflicts of interest.

\section{Funding statement}

The authors received no financial support for the research, authorship and/or publication of this article.

\section{Declaration of authorship}

RKD, AB, NDP and IKF conceived and designed the study; RKD, AB, NDP, IKF, TS, VTD, MN, ZC, ZBM, TL, DT, ZV and IT collected, analysed and interpreted data; all authors made the manuscript; RKD, AB, NDP and SP have critically revised the manuscript for important intellectual content; all authors approved the submitted version; all authors agree that they will be responsible for all aspects of the work.

\section{References}

1. Liang W, Guan W, and Chen R, et al (2020) Cancer patients in SARS-CoV-2 infection: a nationwide analysis in China Lancet Oncol 21(3) 335-337 https://doi.org/10.1016/S1470-2045(20)30096-6 PMID: 32066541 PMCID: 7159000

2. Lee L, Cazier J, and Starkey T, et al (2020) COVID-19 mortality in patients with cancer on chemotherapy or other anticancer treatments: a prospective cohort study Lancet 395(10241) 1919-1926 https://doi.org/10.1016/S0140-6736(20)31173-9 PMID: 32473682 PMCID: 7255715

3. Miyashita H, Mikami T, and Chopra N, et al (2020) Do patients with cancer have a poorer prognosis of COVID-19? An experience in New York City Ann Oncol 31(8) 1088-1089 https://doi.org/10.1016/j.annonc.2020.04.006 PMID: 32330541 PMCID: 7172785

4. Salunke AA, Nandy K, and Pathak SK, et al (2020) Impact of COVID-19 in cancer patients on severity of disease and fatal outcomes: a systematic review and meta-analysis Diabetes Metab Syndr 14(5) 1431-1437 https://doi.org/10.1016/j.dsx.2020.07.037 PMID: 32755847 PMCID: 7833306

5. Dai M, Liu D, and Liu M, et al (2020) Patients with cancer appear more vulnerable to SARS-COV-2: a multi-center study during the COVID-19 outbreak Cancer Discov 10(6) 783-791 https://doi.org/10.1158/2159-8290.cd-20-0422 PMID: 32345594 PMCID: 7309152 
6. Croatian Bureau of Statistics-Republic of Croatia (2020) Dzs.hr [https://www.dzs.hr] Date accessed: 21/10/20

7. Croatian Bureau of Statistics-Republic of Croatia (2020) Dzs.hr [https://www.dzs.hr/Hrv_Eng/ljetopis/2018/sljh2018.pdf] Date accessed: 10/10/20

8. Croatian Institute for Public Health (2020) Coronavirus - latest recommendations Hzjz.hr. Koronavirus - najnovije preporuke [https:// www.hzjz.hr/sluzba-epidemiologija-zarazne-bolesti/koronavirus-najnovije- preporuke/] Date accessed: 10/06/20

9. Jazieh A, Akbulut H, and Curigliano G, et al (2020) Impact of the COVID-19 pandemic on cancer care: a global collaborative study JCO Glob Oncol 6 1428-1438 https://doi.org/10.1200/GO.20.00351 PMID: 32986516 PMCID: 7529504

10. Al-Quteimat OM and Amer AM (2020) The impact of the COVID-19 pandemic on cancer patients Am J Clin Oncol 43(6) 452-455 https://doi.org/10.1097/COC.0000000000000712 PMID: 32304435 PMCID: 7188063

11. Coronavirus disease (COVID-19)-World Health Organization (2020) [https://www.who.int/emergencies/diseases/novel-coronavirus-2019] Date accessed: 10 June 2020

12. Johns Hopkins Coronavirus Resource Centre (2020) COVID-19 Map [https://coronavirus.jhu.edu/map.html] Date accessed: 26/06/20

13. Coronavirus Government Response Tracker. Bsg.ox.ac.uk (2020) [https://www.bsg.ox.ac.uk/research/research-projects/coronavirusgovernment-response-tracker] Date accessed: 26/06/20

14. Ecdc.europa.eu (2020) Homepage|European centre for disease prevention and control COVID-19 pandemic [https://www.ecdc.europa. eu/en/covid-19-pandemic] Date accessed: 25 /06/20

15. Croatian Bureau of Statistics-Republic of Croatia (2020) Basic characteristics of household consumption, 2017. Dzs.hr [https://www. dzs.hr/Hrv_Eng/publication/2018/14-01-02_01_2018.htm] Date accessed: 10/07/20

16. Ghosh J, Ganguly S, and Mondal D, et al (2020) Perspective of oncology patients during COVID-19 pandemic: a prospective observational study from India JCO Glob Oncol 6 844-851 https://doi.org/10.1200/GO.20.00172 PMID: 32552110 PMCID: 7328097

17. de Joode K, Dumoulin D, and Engelen V, et al (2020) Impact of the coronavirus disease 2019 pandemic on cancer treatment: the patients' perspective Eur J Cancer 136 132-139 https://doi.org/10.1016/j.ejca.2020.06.019 PMID: 32683273 PMCID: 7334940

18. Quaquarini E, Saltalamacchia G, and Presti D, et al (2020) Impact of COVID-19 outbreak on cancer patient care and treatment: data from an outpatient oncology clinic in Lombardy (Italy) Cancers 12(10) 2941 https://doi.org/10.3390/cancers12102941 PMCID: 7601200

19. Patt D, Gordan L, and Diaz M, et al (2020) Impact of COVID-19 on cancer care: how the pandemic is delaying cancer diagnosis and treatment for American seniors JCO Clin Cancer Inform 4 1059-1071 https://doi.org/10.1200/CCI.20.00134 PMID: 33253013 PMCID: 7713534

20. Moujaess E, Kourie HR, and Ghosn M (2020) Cancer patients and research during COVID-19 pandemic: a systematic review of current evidence Crit Rev Oncol Haematol 150102972 https://doi.org/10.1016/j.critrevonc.2020.102972 